\title{
Characterization of a Glow Discharge Plasma as a Function of Sampling Orifice Potential
}

\author{
W. S. Taylor, J. G. Dulak, and S. N. Ketkar \\ Extrel Corporation, Pittsburgh, Pennsylvania, USA
}

\begin{abstract}
Plasma diagnostic studies have been carried out on the discharge source of a commercial glow discharge quadrupole inass spectrometer. Plasma parameters were determined using an electrostatic probe with the objective of determining the dependence (if any) of these parameters on the voltage placed on an auxiliary electrode immersed in the plasma. The biased clectrode utilized in this study was the sampling orifice element itself. Our results indicate that, for positive orifice voltages with respect to the grounded anode, variations in the plasma potential and ion energy can be correlated directly to the bias placed on the sampling orifice. The dependence of the electron temperature on this parameter is observed to be more complex in nature, and electron number densities show little significant variation with respect to sampling orifice bias. In addition, increased orifice voltages result in an increase in the ion signal intensity measured with the mass spertrometer. Based on the results obtained here, we feel that this increase is due primarily to an increase in ion transmission to the quadrupole arising from the increased ion energy. (J Am Sac Mass Spectrom 1990, 1 , 448-454)
\end{abstract}

$\mathrm{T}$ The use of glow discharges as ion sources for mass spectrometry is becoming increasingly popular. As a method of solids analysis, glow discharge mass spectrometry (GDMS) has the advantage of providing a stable ion signal, with the additional benefit of exhibiting minimal matrix effects. 'l'he development of GDMS as an analytical technique was recently reviewed by Harrison and co-workers $[1,2]$, as well as by Hess and Marcus [3]. These reviews well illustrate the general utility of this method of analysis. Glow discharges have been utilized successfully in conjunction with both magnetic sector $[4,5]$ and quadrupole mass spectrometers [5-15], with each technique presenting its own advantages. Magnetic sector instruments possess good sensitivity and extreme resolving power, while quadrupole devices provide economy and ease of use. Recent developments in glow discharge quadrupole mass spectrometry (GDQMS) include the use of an $\mathbf{r f}$ discharge source for the analysis of nonconducting samples [11], and the elimination of molecular interferences via collision-induced dissociation [13-15]. In general, data obtained on both magnetic sector and quadrupole glow discharge instruments agree well, confirming that, as we would expect, the mass spectrometer has no effect on the processes occurring in the glow discharge source.

A schematic of the glow discharge showing the mechanisms occurring therein is given in Figure 1 [1]. Upon examination of this figure we see that the sam-

Address reprint requests to W. S. Taylor, Extrel Corporation, 575 Epsilon Drive, Pittsburgh, PA 15238. ple to be analyzed is in fact the cathode. The anode is usually the discharge cell housing, which is at ground potential. When a sufficient potential is placed across the cathode and the anode, the working gas, which is most often argon at approximately 1 torr, is ionized. Positive ions of the discharge gas are then accelerated across a region of large potential drop near the cathode known as the cathode fall, and bombard the surface of the cathode, thereby sputtering cathode material off the surface. Secondary ions produced in this sputtering step are trapped by the large field gradient near the cathode and are redeposited onto the surface. Sputtered neutral species, on the other hand, are not subject to the effects of this field gradient and diffuse out into the negative glow region of the discharge. Once in the negative glow, these sputtered neutrals are ionized by a number of mechanisms, of which the predominant two are electron impact ionization (EI) and Penning ionization by Ar metastables. Of these two, the latter has been shown to be the primary mechanism for the ionization of sputtered cathode species $[16,17]$. Sampling of the analyte ions is carried out via a small orifice immersed in the negative glow.

As in any analytical technique, the signal-to-noise ratio must be maximized in order to optimize the performance of the instrument and obtain good sensitivity. With respect to GDQMS this means that ion production and transmission to the quadrupole mass filter be maximized. Harrison and Bentz [7] noted that the application of a secondary high voltage, low current discharge located near the sampling aperture resulted in an increase in the analyte signal intensity in their 


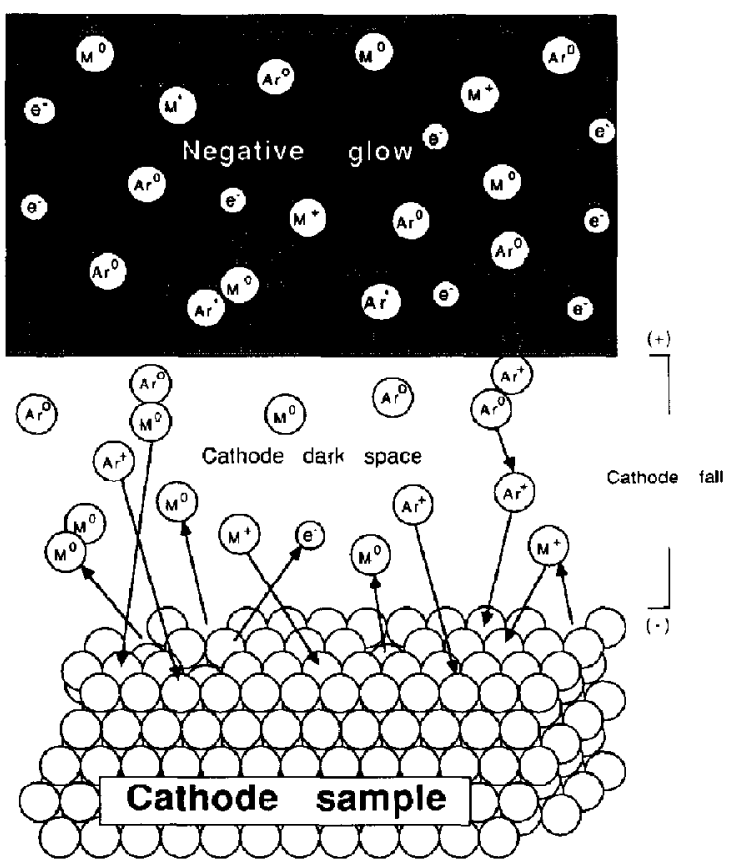

Figure 1. Processes occurring in the glow discharge.

GDQMS. Their conclusion was that this secondary discharge served to further ionize neutral sputtered species. In later work ${ }_{r}$ Jakubowski et al. [18] asserted that the primary effect of this secondary discharge was that of modifying the potential distribution near the sampling orifice rather than enhancing ionization of analyte species. They showed that by manipulating the potential on the sampling orifice plate, effects similar to those noted by Harrison could be achieved. We also noted a dependence of the analyte ion signal on the bias placed on the electrically isolated sampling orifice element in our prototype GDQMS. More specifically, we note an increase in signal as the bias on this component becomes more positive with respect to ground potential. To attempt to elucidate the nature of this signal enhancement, we have carried out Langmuir probe studies on our glow discharge source in order to characterize the plasma as a function of the potential on the sampling orifice element. Plasma parameters such as plasma potential $\left(V_{p}\right)$, electron temperature $\left(T_{e}\right)$, and electron number density $\left(N_{e}\right)$ were calculated at various orifice potentials $\left(V_{0}\right)$ with the ultimate objective of exploiting any observed effects such that instrument performance is optimized.

\section{Experimental}

A schematic of the EXT 1000 glow discharge quadrupole mass spectrometer (Extrel Corp., Pittsburgh, PA) is given in Figure 2. The details of this instrument have been described previously [12]. The only modification made to this instrument for the pur-

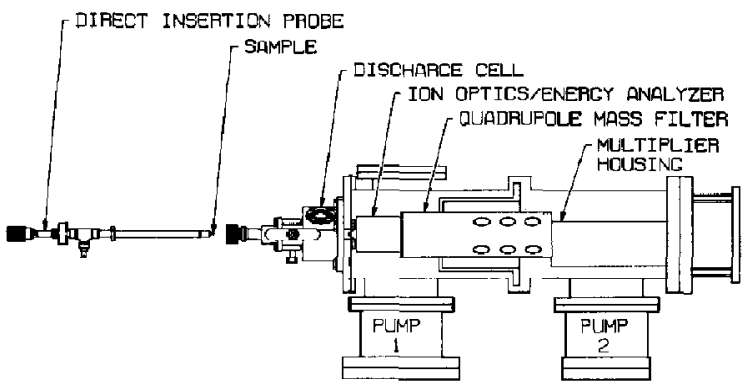

Figure 2. Schematic of the glow discharge quadrupole mass spectrometer used in this study.

poses of this study was the addition of the Langmuir probe into the discharge cell. The experimental set-up used for the Langmuir probe measurements is illustrated in Figure 3. Langmuir probes were fashioned out of tungsten wire $0.127 \mathrm{~mm}$ in diameter. Except for that portion of the probe which was to be immersed in the plasma, the wire was encapsulated in a $6.35-\mathrm{mm}$ O.D. glass tube. The exposed portions of the probes varied in length from $2.4 \mathrm{~mm}$ to $6.9 \mathrm{~mm}$. The glass portions of these probes were fashioned such that the exposed wire was shielded from deposition of sputtered materials by a reentrant seal. This serves to prevent the probe itself from becoming shorted to the plated material that accumulates on the glass sheath during the course of the experiment [19]. Examination of these probes subsequent to the experiment confirmed that no deposition had occurred within the reentrant. During the experiment, the probe was positioned $4 \mathrm{~mm}$ from the sampling orifice, which itself was located 8 mun from the end of the cathode. Voltage was applied to the probe over a range of -24 to $+24 \mathrm{~V}$ using a Hewlett-Packard 6236A power supply (North Hollywood, CA). This power supply was referenced to the anode of the discharge, which was at ground po-

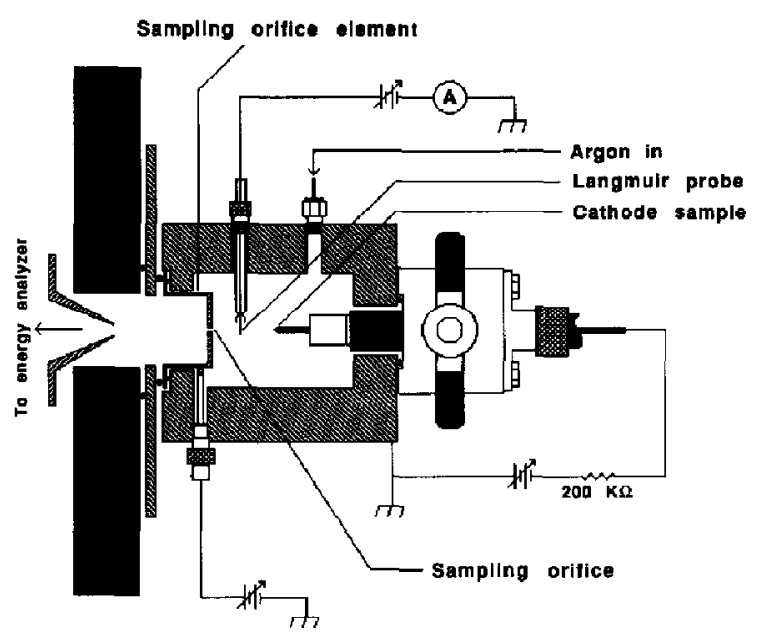

Figure 3. Detail of the discharge region of the glow discharge mass spectrometer showing the Langmuir probe. 


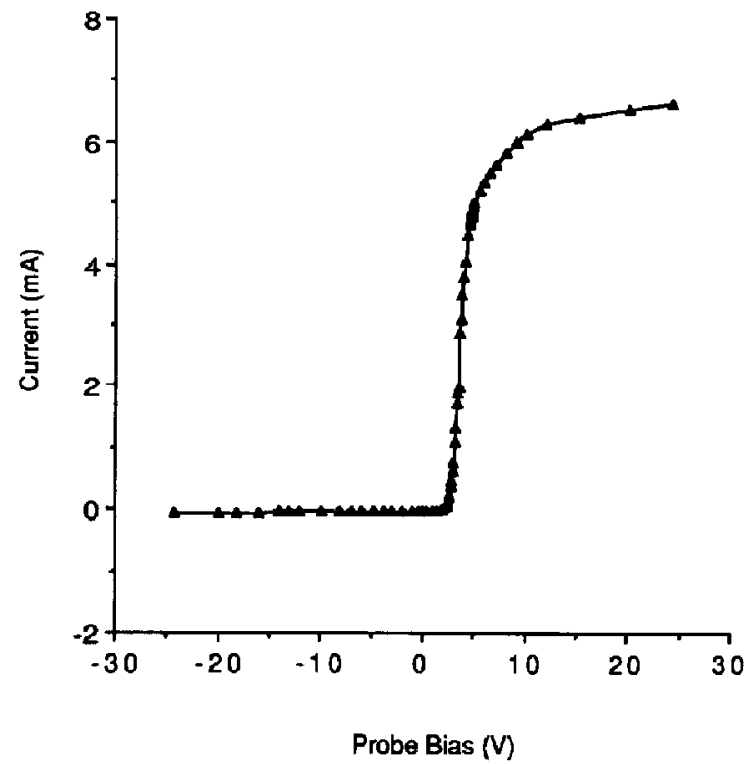

Figure 4. Typical I-V characteristic curve obtained in this study illustrating the specific case in which $2 \mathrm{~V}$ was applied to the sampling orifice element.

tential. Current collected by the Langmuir probe was measured with an ammeter. The voltage applied to the sampling orifice element was varied between -5.24 and $13.05 \mathrm{~V}$ with respect to ground potential. The cathode of the discharge was a 303 stainless steel pin 12.7 $\mathrm{mm}$ in length and $1.5 \mathrm{~mm}$ in diameter. The discharge voltage was set at approximately $1500 \mathrm{~V}$, with a current of $4 \mathrm{~mA}$. A $200 \mathrm{k} \Omega$ ballast resistor was placed in series with the discharge. Ultrahigh purity argon was used as the working gas of the discharge and was maintained at approximately $700 \mathrm{mtorr}$ in the discharge cell.

\section{Determination of Plasma Parameters}

With the use of the Langmuir probe, I-V characteristic curves, such as the one shown in Figure 4, were generated at several sampling orifice potentials $\left(V_{0}\right)$. As is explained by Cox et al. [20], the I-V characteristic curve can be divided into three distinct regions, which are expressed mathematically below. These regions are: $(A)$ the electron saturation region; $(B)$ the electron retardation region; and $(C)$ the ion saturation region. These three regions of the curve are characterized by the sign and magnitude of the probe potential, $\mathrm{V}$, with respect to the plasma potential, $\mathrm{V}_{\mathrm{p}}$ :

(A) $\mathrm{V}-\mathrm{V}_{\mathrm{p}}>0$

(B) $V-V_{p}<0$

(C) $\mathrm{V}-\mathrm{V}_{\mathrm{p}}<0$ and $\left|\mathrm{V}-\mathrm{V}_{\mathrm{p}}\right| \gg \mathrm{kT}_{\mathrm{e}} / \mathrm{e}$

where $\mathrm{V}$ is the voltage placed on the Langmuir probe, $\mathrm{k}$ is the Boltzmann's constant, and $\mathrm{e}$ is the electronic charge. The plasma potential at the location of the probe was derived by determining the maximum of the first derivative curve of the I-V characteristic curve. Calculation of the electron temperature and electron number density was carried out with the use of the equations and methods outlined below, which assume that the kinetic energy distributions of ions and electrons in the plasma are Maxwellian in nature. Upon making this assumption, we can write the following expression for the electron current [21].

$$
\mathrm{I}_{\mathrm{e}}=\mathrm{AeN}_{\mathrm{e}}\left[\left(\mathrm{kT}_{\mathrm{e}}\right) /\left(2 \pi \mathrm{m}_{\mathrm{e}}\right)\right]^{1 / 2} \exp \left[-\left[\mathrm{e}\left(\mathrm{V}_{\mathrm{p}}-\mathrm{V}\right)\right] /\left(\mathrm{kT}_{\mathrm{e}}\right)\right]
$$

In this equation, A represents the surface area of the probe and $m_{e}$ is the mass of an electron. We can obtain $I_{e}$ by subtracting the ion current $I_{+}$from the total current in the electron retardation region. The ion current in this region is obtained by linear extrapolation of the ion saturation portion of the I-V characteristic curve. By then taking the natural logarithm of eq $\mathbf{1}$, we obtain a linear relationship, the slope of which is represented by $e / k T_{e}$. By applying this equation to the electron retardation region of the I-V characteristic, $T_{e}$ can be determined. If we then take the electron current at the point where $\mathrm{V}=\mathrm{V}_{\mathrm{p}}$, designated by $\mathrm{I}_{0}$, eq 1 reduces to:

$$
I_{0}=\operatorname{AeN}_{\mathrm{e}}\left[\left(k \mathrm{~T}_{\mathrm{e}}\right) /\left(2 \pi \mathrm{m}_{\mathrm{e}}\right)\right]^{1 / 2}
$$

From eq 2 it is then possible to derive the electron number density, $\mathrm{N}_{\mathrm{e}}$.

Thus we see that it is possible to obtain all the pertinent plasma parameters from the set of data represented in the I-V characteristic curve. It is necessary, however, to view these interpretations with caution because probe measurements can be fraught with errors $[19,21,22]$, not the least of which arises from the fact that the probe surface area changes during the course of the experiment due to the deposition of cathode materials onto the probe. This can cause problems in the calculation of plasma parameters that involve the surface area of the probe such as $N_{e}$, but will not have an effect on the determination of $V_{p}$ and $T_{e}$, neither of which are dependent on probe surface area. The independence of $V_{p}$ on probe area is fortuitous in the case of these experiments because as will be seen, the most significant effects of sampling orifice potential on the plasma are those related to plasma potential.

\section{Results and Discussion}

\section{Plasma Potential}

Given in Figure 5 are probe characteristics for orifice potentials from 0 to $20 \mathrm{~V}$ in increments of $5 \mathrm{~V}$. In this plot, the vertical scale is the same for each characteristic curve. We immediately note two distinct features of these curves. First, it is apparent that as the sampling 
orifice bias becomes more positive, the characteristic curves are shifted in the positive direction. We also see that the amount that each curve is shifted relative to the previous curve appears to be the same in each case. More will be said about this later. We also observe that the curves become more symmetrical as the orifice bias increases. This is particularly apparent if we compare the curve at an orifice bias of $0 \mathrm{~V}$ to the curves obtained with higher orifice potentials. The characteristic curve with a grounded sampling orifice displays the typical sharp transition through the electron retardation region followed by a much gentler transition into the electron saturation region. This latter transition becomes much less pronounced in the other three curves. The exact nature of this behavior is still in question, but has been noted in other glow discharge sources [23].

If we plot plasma potential as a function of orifice bias we can immediately observe a distinct correlation between these two parameters. This is illustrated in Figure 6 where the plasma potential for several values of $V_{0}$ is presented. There is an apparent break in the curve that occurs around $0 \mathrm{~V}$, but there is a direct linear correlation between $V_{o}$ and $V_{p}$ for values of $\mathrm{V}_{0}$ above $0 \mathrm{~V}$. This fact is demonstrated in Figure 6 , where we have carried out a linear regression analysis on the data representing positive orifice potentials only. This best fit has a slope of unity with an intercept of $1.9 \mathrm{~V}$, which, based on the available data and experiments carried out by other researchers [24], is the approximate plasma potential for $\mathrm{V}_{\mathrm{o}} \leq 0 \mathrm{~V}$.

$$
V_{p}=1.0\left(V_{o}\right)+1.9
$$

As indicated in Figure 6, at positive orifice potentials, $V_{p}$ can be related to $V_{o}$ through eq 3 (for this particular location in the plasma), but that at orifice potentials

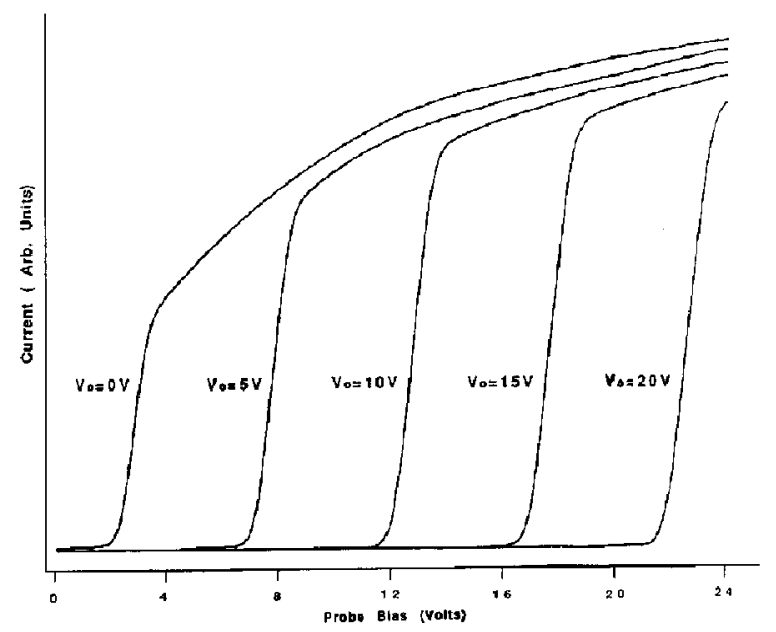

Figure 5. Variation of I-V characteristics as a function of sampling orifice bias.

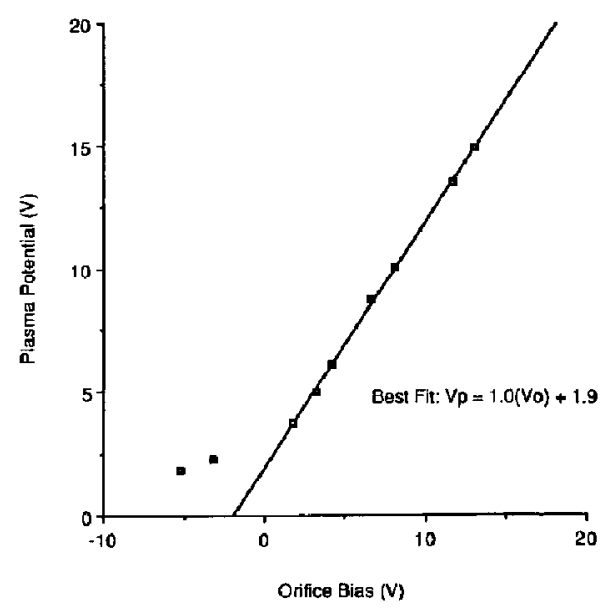

Figure 6. Linear dependence of plasma potential on the bias placed on the sampling orifice element for orifice voltages greater than $\mathrm{OV}$

of $0 \mathrm{~V}$ and below, this relationship no longer holds. Furthermore, it is reasonable to assume that similar linear relationships relating these two parameters exist for other locations in negative glow region that differ only in the value of the intercept. Similar variations of $V_{p}$ with respect to $V_{o}$ were noted previously by Coburn and Kay [24] in an If glow discharge source where behavior similar to that depicted in Figure 6 was observed. Their observations as well as those noted in this work agree with the assertion by Chapman [21] that $V_{p}$ must be the most positive potential in sputtering discharges.

\section{Ion Energy}

The EXT 1000 is equipped with a Bessel Box energy analyzer, which makes it possible to obtain representations of the ion energy distributions at the point of sampling as a function of $V_{0}$. A Bessel Box is simply a metal cylinder to which a potential is applied such that a retarding electric field is established within it. On either end of this cylinder are electrostatic bandpass lenses. A neutral stop inside the Bessel Box is positioned in such a way that there is no direct path through the device. Ions entering the cylinder of the energy analyzer with the appropriate translational energy are severely retarded, and are then reaccelerated upon emergence. Ions with excessive translational energy relative to the potential on the cylinder collide with the walls, and ions with too little energy are rejected. The name "Bessel Box" derives from the fact that a series of Bessel functions is used in solving for the ion trajectories through the energy analyzer. Ion energy data were obtained using this device at several sampling orifice potentials between 0 and $10 \mathrm{~V}$. These curves are illustrated in Figure 7, and were obtained by observing the peak intensity of the isotope 


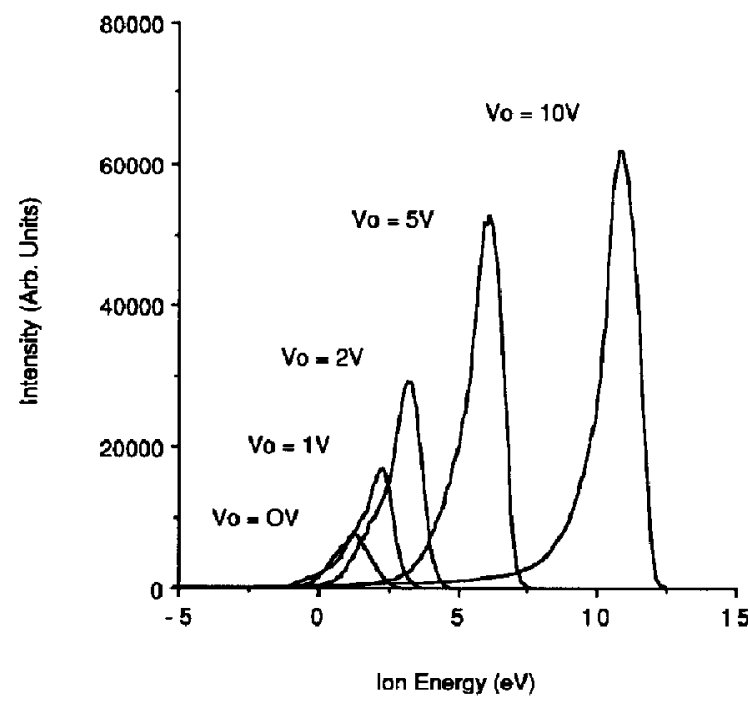

Figure 7. Ion energy distributions of ${ }^{56} \mathrm{Fe}^{+}$ions as a function of bias placed on the sampling orifice element.

of iron occurring at $56 \mathrm{u}$ as a function of the cylinder potential on the energy analyzer. The data were then calibrated by adding the amount of voltage required to eliminate the signal entirely at the entrance to the quadrupole mass filter. This stopping voltage was determined by manipulating the potential of the $\mathrm{z}$ axis of the quadrupole. The values reported as ion energy on the abscissa of Figure 7 are simply the sum of the Bessel Box cylinder potential and the stopping potential. The fact that a small offset potential must be added to the voltage applied to the cylinder can be understood by noting that, for a given cylinder voltage, ions with slightly more energy are subject to maximum transmission through the device [25].

There are some salient features of these curves that merit discussion. We first note that, as in the case of the plasma potential, the ion energy curves exhibit a dependence upon the value of $V_{0}$. In order to investigate this relationship further, the maxima of several of these energy distribution curves at various values of $V_{0}$ were determined. This peak energy is then represented as a function of orifice bias. Data of this nature are given in Figure 8 for $V_{0}>0 \mathrm{~V}$. Here, as in the case of the plasma potential, we observe a linear correlation between the ion energy, $E_{i}$, and the orifice potential with the best fit given by:

$$
E_{i}=0.95\left(V_{0}\right)+0.67
$$

At a fixed quadrupole bias, the potential energy of the ions relative to the quadrupole at the instant of their formation is defined by the plasma potential at that location. As $V_{o}$ was shown to dictate the value of $V_{p}$, eq 4 actually demonstrates that $E_{i}$ is defined by $V_{0}$ also, which is a fact that has long been recognized by researchers examining sputter deposition processes $[19,24]$. By solving eq 3 for $V_{0}$ in terms of $V_{p}$ and then substituting for $V_{0}$ in eq 4 , we obtain the following relationship for $E_{i}$ in terms of $V_{p}$

$$
E_{i}=0.95\left(V_{p}\right)-1.2
$$

for values of $V_{0}$ where eq 3 holds true (i.e., $V_{0}>0$ ). The difference of approximately $1 \mathrm{~V}$ between $\mathrm{E}_{\mathrm{i}}$ and $V_{p}$, as is illustrated by eq 5 , suggests that the ions that are ultimately drawn out of the discharge cell are in fact formed much closer to the sampling orifice than the location of the Langmuir probe.

The shape of these ion energy distribution curves is very reminiscent of those of sputtered species determined in previous work with a $90^{\circ}$ electrostatic energy analyzer [24], indicating that our results are consistent in this regard with those obtained with other glow discharge sources. The fact that the low energy tails of several of the curves given in Figure 7 extend into negative values suggests some error associated with the absolute positions of the ion energy distributions, which would result in a different value for the intercept in eq 5 . This effect has also been noted in previous work [7] where a Bessel Box was used for energy analysis. However, because we did in fact correct our ion energy values with a stopping potential, we feel that this error is small.

Referring to Figure 7, it is apparent that signal intensity shows a marked dependence on $V_{0}$. Upon a

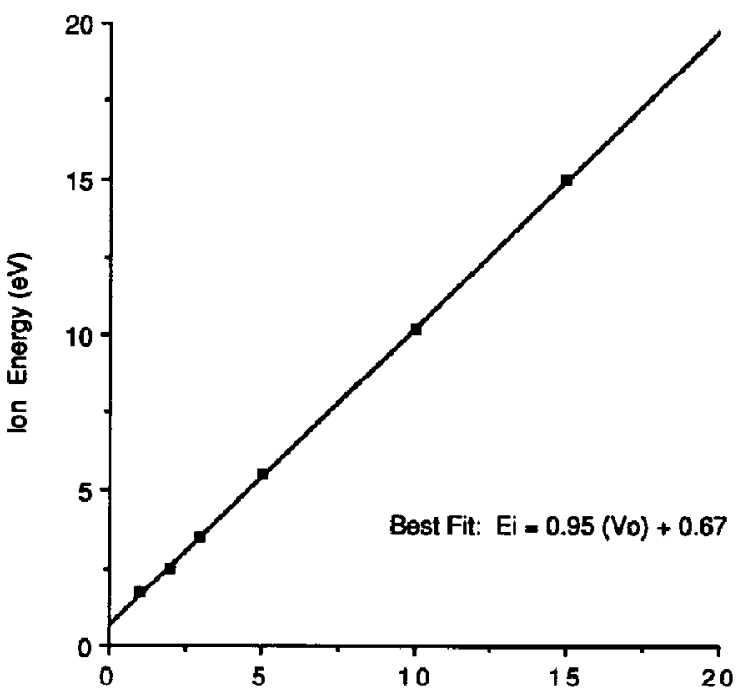

Orifice Bias $(N)$

Figure 8. Linear dependence of ion energy on the bias placed on the sampling orifice element for orifice voltages greater than $0 \mathrm{~V}$. 
comparison of the energy distribution curves for orifice potentials of 0 and $10 \mathrm{~V}$, we see that the absolute signal intensity is enhanced at $\mathrm{V}_{0}=10 \mathrm{~V}$ by roughly a factor of 8 . The variation in the maxima of these distributions is in fact observed in the mass spectra obtained with the EXT 1000, and thus the bias on the sampling orifice is "tuned" as if it were another ion optic element for maximum signal. It must be noted, however, that at a fixed pole bias, the user cannot simply adjust $V_{0}$ to a very high positive value in order to obtain maximum sensitivity. This is because of the inherent restrictions placed on the ion energy by the quadrupole mass filter itself. If the translational energy of the ions passing through the quadrupole mass filter becomes too high, the quadrupole loses resolving power because the ions do not experience a residence time in the quadrupole sufficient for mass analysis. The result of this phenomenon is that the peak shapes become distorted and are not useful for analytical methods. Thus, there is a limit to the amount of signal enhancement that may be obtained via adjustment of the orifice bias. In practice, we have found that analyte ion signals can be optimized with regard to absolute intensity and peak shape with an orifice bias between $2 \mathrm{~V}$ and $5 \mathrm{~V}$ with respect to ground.

\section{Electron Temperature}

In order to further investigate the effects of the potential applied to the sampling orifice on plasma parameters, the $T_{e}$ was calculated as described previously by obtaining the slope of the plot of $\ln \left(\mathrm{I}_{e}\right)$ vs. probe bias. Typical values for correlation coefficients from these regression analyses are on the order of 0.999 , indicating adherence to the Maxwellian assumption. Not surprisingly, given the previously noted observations regarding the behavior of $V_{p}$ with respect to $V_{o}$, the linear regions of these plots were observed to shift to higher voltages as $V_{0}$ was increased. The results of these calculations are summarized in Figure 9. These values for the electron temperature are consistent with those obtained in previous experiments on dc glow discharges using similar devices $[23,26]$. As is evident upon examination of Figure $9, T_{e}$ does indeed trend with $V_{0}$. The data range from $2400 \mathrm{~K}$ to $5050 \mathrm{~K}$, which corresponds to a total difference in these observations of approximately $0.2 \mathrm{eV}$. If indeed the kinetic energy distributions of the electrons can be described as Maxwellian, this increase in electron temperature corresponds to a shift in the energy distribution toward higher energies. A possible explanation for this observation may be that the discharge experiences some degree of localization between the cathode and the sampling orifice element. As the sampling orifice becomes more positively biased, the electron current to this element increases. Because the potential of the sampling orifice is positive with respect to the cell body (ground potential), the discharge power is increased, resulting in a slightly

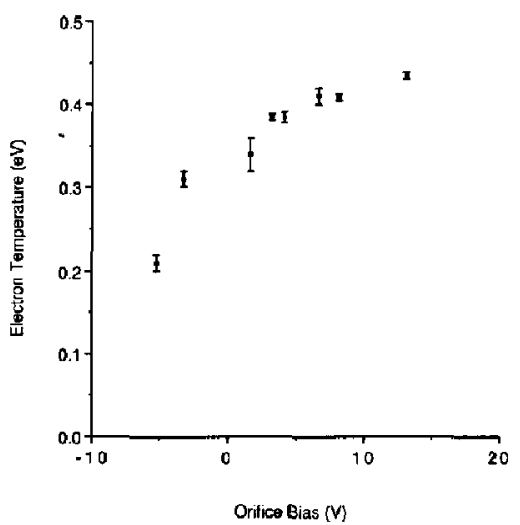

Figure 9. Variation of electron temperature as a function of sampling orifice bias.

higher $T_{e}$. As was mentioned previously, Penning ionization via Ar metastable species is thought to be an important mechanism for ionization of sputtered neutrals. The lowest Ar metastable state occurs at 11.55 $\mathrm{eV}$. The shift in the electron energy distribution described by the increase in $T_{e}$ shown in Figure 9 results in an increase of several orders of magnitude in the fraction of electrons possessing energies at and above this threshold energy. While we observe an increase in analyte signal intensity, it is only an increase of approximately a factor of 8 . This apparent inconsistency suggests the existence of some other contributing factor(s) that were not examined during the course of this work and indicates the need for experiments to determine the dependence of the Ar metastable populations on voltages placed on auxiliary electrodes immersed in the plasma. One possibility that must be considcred is that the Maxwellian assumption is not valid. This is thought to be true with respect to the high energy end of the electron energy distribution in glow discharges because of the existence of several mechanisms that deplete high energy electrons [21]. As we have seen, however, our data indicate Maxwellian behavior in this case. This is in agreement with arguments outlined by Chapman [21] that assert that energy is being dissipated in the glow by electrons from the cathode sheath, resulting in a shift in the electron energy distribution back toward Maxwellian.

\section{Electron Number Density}

Electron number densities at several values of $V_{0}$ were calculated using the method described above. These data are presented in Figure 10, where an estimate of the amount of error introduced by changes in probe surface area is included. We first note that the values obtained here for $N_{e}$ are not unreasonable for the negative glow region of the discharge. Furthermore, examination of these data reveals that if any dependence of 


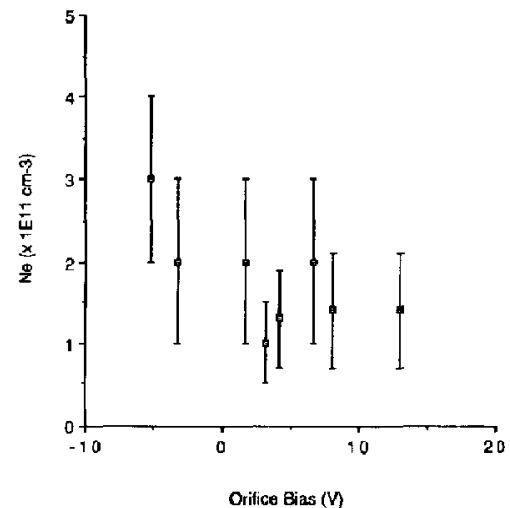

Figure 10. Behavior of electron number density as a function of sampling orifice bias.

electron number density on orifice bias exists, this dependence is weak. This suggests that, within the limits of the error of this measurement, increased orifice bias does not result in a significant increase in the degree of ionization occurring in the plasma.

\section{Summary}

The observations obtained in the course of these studies indicate that increased orifice bias has a marked effect on the plasma potential, and by changing the plasma potential, the ion energy relative to the quadrupole mass filter can be manipulated. In addition, a slight dependence of electron temperature on orifice bias was observed, while no detectable relationship between electron number density and orifice bias was noted. This would suggest that the increase in analyte signal intensity with $V_{o}$ is due primarily to an improvement in the transmission of the ions to the quadrupole rather than an enhancement of any ionization mechanism(s). The behavior of plasma potential demonstrated here with respect to $V_{o}$ is quite consistent with that observed in previous work [24], suggesting that similar results might be obtained with any secondary electrode immersed in the plasma. This indicates to us that it might be possible to optimize the geometry and positioning of this secondary electrode such that ever greater signal enhancenents might be realized. The effect of this electrode is much the same as that of the ion region in the $\mathrm{El}$ source in most commercially available mass spectrometers, and is utilized in the same manner on our instrument during the tuning procedure. By treating the sampling aperture bias as another tuning element, it is possible to utilize the observed signal enhancements as a means to further increase sensitivity. Because the detection limits of quadrupole based glow discharge mass spectrometers are significantly higher than those of magnetic sector instruments, this is an area where improvements in instrument performance are of great concern.

\section{References}

1. Harrison, W. W.; Bentz, B. L. Prog. Anal. Spectrosc. 1988, 11, 53.

2. Harrison, W. W.; Hess, K. R.; Marcus, R. K.; King, F. L. Anal. Chem. 1986, 58, 341A.

3. Hess, K. R.; Marcus, R. K. Spectroscopy 1987, 2, 24.

4. Sanderson, N. E.; Hall, E.; Clark, I.; Charalambous, P.; Hall, D. Mikrochim. Acta 1987, 1, 275.

5. Grasserbauer, M. Mikrochim. Acta 1987, 1, 291.

6. Bentz, B. L.; Bruhn, C. G.; Harrison, W. W. Int. J. Mass Spec. Ion Phys. 1978, 28, 409.

7. Harrison, W. W.; Bentz B. L. Anal. Chem. 1979, 51, 1853.

8. Jakubowski, N.; Stuewer, D.; Vieth, W. Fresenius Z. Anal. Chem. 1988, 331, 145.

9. Jakubowski, N.; Stuewer, D.; Vieth, W. Anal. Chem. 1987, $59,1825$.

10. Marcus, R. K.; Fang, D.; Kolar, W.; Winchester, M. R. Presented at the Colloquium Sectroscopicum Internationale XXV, Toronto, Canada, June 21-26, 1987.

11. Duckworth, D. C.; Marcus, R. K. Anal. Chem. 1989, 61, 1879.

12. Taylor, W. S.; Dulak, J. G. Spestroscapy 1989, 4, 41

13. King, F. L.; McCormack, A. L.; Harrison, W. W. J. Anal. At. Spectron. 1988, 3, 883.

14. King, F. L.; Harrison, W. W. Int. J. Mass Spec. Ian Prac. 1989, 89, 171.

15. Duckworth, D. C.; Marcus, R. K. Appl. Spectrosc., 1990, 44, 649.

16. Hess, K. R.; Harrison, W. W. Anal. Chem. 1988, 60, 691.

17. Smith, R. L.; Serxner, D.; Hess, K. R. Anal. Chem. 1989, 61, 1103.

18. Jakubowski, N.; Stuewer, D.; Toelg, G. Int. J. Mass Spec. Ion Proc. 1986, 71, 183.

19. Clements, R. M. J. Vac. Sci. Technol. 1978, 15, 193.

20. Cox, T. I.; Deshmukh, V. G. I.; Hope, D. A. O.; Hydes, A. J.; Braithwaite, N. St. J.; Benjamin, N. M. P. J. Phys. D: Appl. Phys. 1987, 20, 820.

21. Chapman, B. N. Glow Discharge Processes; Wiley: New York, 1480 .

22. Chen, F. F. In Plasma Diagnostic Techniques, Huddlestone, R. H.; Leonard, S. L., Eds.; Academic: New York, 1965; Chap. 4.

23. Fang, D.; Marcus, R. K. Spectrochim. Acta B, in press.

24. Coburn, J. W.; Kay, E. J. Appl. Phys. 1972, 43, 4965.

25. Craig, J. H. Jr.; Durrer, W. G. J. Vac. Sci. Technol. A 1989, 7, 3337.

26. Esar, E.; Ogilvie, R. E.; Taylor, K. A. J. Vac. Sci. Techrol. 1978, $15,199$. 